\title{
LYUBEZNIK NUMBERS IN MIXED CHARACTERISTIC
}

\author{
Luis NúÑEZ-Betancourt AND Emily E. WitT
}

\begin{abstract}
We define a family of invariants associated to any local ring whose residue field has prime characteristic; in particular, this includes those of mixed characteristic. These Lyubeznik numbers in mixed characteristic are defined via a surjection from an unramified regular local ring of mixed characteristic. As is true for the Lyubeznik numbers, the "highest-index" Lyubeznik number in mixed characteristic is a well-defined notion. These new invariants and the original Lyubeznik numbers coincide for certain local rings of equal characteristic $p>0$; e.g., for Cohen-Macaulay rings and rings of dimension at most two. However, we provide an example where they differ.
\end{abstract}

\section{Introduction}

1.1. Background. For an ideal $I$ of a regular local ring $S$ of equal characteristic $p>$ 0 , Huneke and Sharp proved that the Bass numbers of the local cohomology modules $H_{I}^{i}(S)$ are finite, and Lyubeznik extended this result to arbitrary equal characteristic using $D$-modules $[12,15]$. He also extended these finiteness results to a larger family of modules, and used them to define a family of integer-valued invariants now called the Lyubeznik numbers.

Let $R$ be a local ring admitting a surjection from a regular local ring $S$ containing a field. If $R$ has residue field $K, S$ has dimension $n$, and $I$ is the kernel of the surjection, then the Lyubeznik number of $R$ with respect to integers $i, j \geq 0$ is defined as $\lambda_{i, j}(R)=\operatorname{dim}_{K} \operatorname{Ext}_{S}^{i}\left(K, H_{I}^{n-j}(S)\right)$. For $R$ any local ring containing a field, defining $\lambda_{i, j}(R)$ to equal $\lambda_{i, j}(\widehat{R})$ extends this definition. Remarkably, these numbers do not depend on the choice of $S$, nor on the surjection [15].

The Lyubeznik numbers encode properties of the ring. For instance, if $R$ has dimension $d, \lambda_{d, d}(R)$ is the number of connected components of the Hochster-Huneke graph of the completion of its strict Henselization [19, 28]. Moreover, these invariants have connections with many areas of mathematics; for example, they have interpretations in terms of singular and étale cohomology $[4,7]$.

1.2. Aim and main results. We define a family of invariants of any local ring whose residue field has prime characteristic, which includes those of mixed characteristic. Employing the Cohen Structure Theorems in mixed characteristic and a Kashiwara equivalence formalized in [21], we give the following definition.

Theorem/Definition (see Theorem 3.5, Definition 3.6). Let $(R, m, K)$ be a local ring such that $K$ has characteristic $p>0$, and let $\widehat{R}$ denote its completion at $m$. By the Cohen Structure Theorems, $\widehat{R}$ admits a surjection $\pi: S \rightarrow \widehat{R}$, where $S$ is an $n$-dimensional unramified regular local ring of mixed characteristic. Let $I=\operatorname{Ker}(\pi)$

Received by the editors May 1, 2013. 
and take $i, j \geq 0$. Then the Lyubeznik number of $R$ in mixed characteristic with respect to $i$ and $j$ is defined as

$$
\widetilde{\lambda}_{i, j}(R)=\operatorname{dim}_{K} \operatorname{Ext}_{S}^{i}\left(K, H_{I}^{n-j}(S)\right) .
$$

This number is finite and depends only on $R, i$, and $j$.

Note that these invariants are finite by $[17,20]$.

Motivated by analogous properties of the Lyubeznik numbers in equal characteristic, we study properties of these invariants (cf. [15, Properties 4.4]; see Section 3). As for the Lyubeznik numbers, the "highest" Lyubeznik number in mixed characteristic is a well-defined notion: if $d=\operatorname{dim}(R)$, then $\widetilde{\lambda}_{i, j}(R)=0$ if $i>d$ or $j>d$ (see Section 4).

For local rings of equal characteristic $p>0$, both the Lyubeznik numbers and the Lyubeznik numbers in mixed characteristic are defined. These families of invariants agree for Cohen-Macaulay rings, and for rings of dimension at most two (see Section 5). However, we give an example of a ring $R$ for which $\widetilde{\lambda}_{i, j}(R) \neq \lambda_{i, j}(R)$ for certain choices of $i, j \geq 0$. To do so, we utilize a computation of Àlvarez Montaner and Vahidi and work of Singh and Walther on Bockstein homomorphisms of local cohomology modules [2, 24] (see Section 6).

\section{Preliminaries}

2.1. $D$-modules. Here, we review some facts about $D$-modules that will be used, and refer the reader to $[3,6]$ for details on these objects of study.

Given commutative rings $R \subseteq S$, the ring of $R$-linear differential operators on $S$, denoted $D(S, R)$, is the (non-commutative) subring of $\operatorname{End}_{R}(S)$ defined inductively as follows: the operators of order zero are the endomorphisms defined by multiplication by some element of $S$, and $\phi \in \operatorname{End}_{R}(S)$ is an operator of order less than or equal to $\ell \geq 1$ if $[\phi, \sigma]=\phi \cdot \sigma-\sigma \cdot \phi$ is an operator of order less than or equal to $\ell-1$ for every $\sigma \in S$. Then $D(S, R)$ is the collection of all operators of order less than or equal to some $\ell \geq 0$. If $S=R \llbracket x_{1}, \ldots, x_{n} \rrbracket$ for some $n>0$, then $D(S, R)=$ $S\left\langle\frac{1}{t !} \frac{\partial^{t}}{\partial x_{i}{ }^{t}} \mid t>0,1 \leq i \leq n\right\rangle[8$, Theorem 16.12.1].

The ring $S$ is naturally a $D(S, R)$-module, and for every $f \in S, S_{f}$ has a natural $D(S, R)$-module structure such that the localization map $S \rightarrow S_{f}$ is a map of $D(S, R)$ modules. This induces a natural $D(S, R)$-module structure on any local cohomology module of the form $H_{I}^{j}(S)$, where $I$ is an ideal of $S$ and $j \geq 0$; moreover, if $I_{1}, \ldots, I_{\ell}$ are ideals of $S$ and $j_{1}, \ldots, j_{\ell} \geq 0$, then all modules of the form $H_{I_{\ell}}^{j_{\ell}} \ldots H_{I_{2}}^{j_{2}} H_{I_{1}}^{j_{1}}(S)$ also have such a structure [15, Example 2.1(iv)]. If $R=K$, a field, and $f \in S=$ $K \llbracket x_{1}, \ldots, x_{n} \rrbracket$, then $S_{f}$ has finite length in the category of $D(S, K)$-modules. Thus, each $H_{I_{\ell}}^{j_{\ell}} \cdots H_{I_{2}}^{j_{2}} H_{I_{1}}^{j_{1}}(S)$ also does [16, Corollary 6].

2.2. A key functor. Lyubeznik utilized the direct image functor from the classical Kashiwara equivalence for smooth varieties to prove that the Lyubeznik numbers are well defined (cf. [15, Lemma 4.3]). In [21, Section 3], the authors further develop the theory of this functor. As we rely greatly on some of these results to show that the Lyubeznik numbers in mixed characteristic are well defined, we include them here for the reader's convenience. Please refer to loc. cit. for details and proofs. 
Fix a Noetherian ring $R$, and let $S=R \llbracket x \rrbracket$. Let $G$ denote the functor from the category of $R$-modules to that of $S$-modules given by $G(-)=(-) \otimes_{R} S_{x} / S$, the direct image functor. For an $R$-module $M$, since $G(M)=M \otimes_{R} S_{x} / S=\bigoplus_{\alpha>0}\left(M \otimes_{R} R x^{-\alpha}\right)$, any nonzero $u \in G(M)$ can be written uniquely in the form $u=m_{1} \otimes x^{-\alpha_{1}}+\cdots+$ $m_{\ell} \otimes x^{-\alpha_{\ell}}$ for some $\ell>0$, and $\alpha_{i}>0$ and $m_{i} \in M, 1 \leq i \leq \ell$.

The functor $G$ defines an equivalence between the category of $R$-modules and that of $D(S, R)$-modules supported on the Zariski-closed subset $\mathcal{V}(x S)$ of $\operatorname{Spec}(S)$. The $D(S, R)$-module structure of $G(M)$ is given by: for $t, \alpha>0$ and $m \in M,\left(\frac{1}{t !} \frac{\partial^{t}}{\partial x^{t}}\right)$. $\left(m \otimes x^{-\alpha}\right)=\left(\begin{array}{c}\alpha+t-1 \\ t\end{array}\right) \cdot\left((-1)^{t} m \otimes x^{-\alpha-t}\right)$. The functor $G$ is also flat, and commutes with taking local cohomology.

For every ideal $I$ of $R$ and every $j \geq 0, G\left(H_{I}^{j}(M)\right) \cong H_{(I, x) S}^{j+1}\left(M \otimes_{R} S\right)$. Moreover, if $R$ is Gorenstein and $P$ is a prime ideal of $R$, then $G\left(E_{R}(R / P)\right)=E_{S}(S /(P, x) S)$. In fact, in this case, $M$ is an injective $R$-module if and only if $G(M)$ is an injective $S$-module.

Remark 2.1. Take $R$-modules $M$ and $N$. Since $R \cong S / x S$, endow $M$ with the natural $S$-module structure via extension of scalars. As $G(N)$ is an $S$-module, $\operatorname{Ext}_{S}^{i}(M, G(N))$ is also an $S$-module. Since it is annihilated by $x S$, it also has an $R$-module structure. As $R$-modules, $\operatorname{Ext}_{S}^{i}(M, G(N))=\operatorname{Ext}_{R}^{i}(M, N)$ for all $i \geq 0$.

\section{Definition and properties}

Lemma 3.1. Let $(R, m, K)$ be a Gorenstein local ring, and let $S=R \llbracket x \rrbracket$. Then for every ideal $I$ of $R$ and all $i, j \geq 0, \operatorname{dim}_{K} \operatorname{Ext}_{S}^{i}\left(K, H_{(I, x) S}^{j+1}(S)\right)=\operatorname{dim}_{K} \operatorname{Ext}_{R}^{i}\left(K, H_{I}^{j}(R)\right)$. Moreover, if $T=R \llbracket x_{1}, \ldots, x_{n} \rrbracket$, then

$$
\operatorname{dim}_{K} \operatorname{Ext}_{T}^{i}\left(K, H_{\left(I, x_{1}, \ldots, x_{n}\right) T}^{j+n}(T)\right)=\operatorname{dim}_{K} \operatorname{Ext}_{R}^{i}\left(K, H_{I}^{j}(R)\right) .
$$

Proof. For $G$ the functor defined in Section 2.2, $G\left(H_{I}^{j}(R)\right)=H_{(I, x) S}^{j+1}(S)$ by $[21$, Lemma 3.9]. Since $R$ is Gorenstein, Remark 2.1 implies that $\operatorname{Ext}_{S}^{i}(M, G(N))=$ $\operatorname{Ext}_{R}^{i}(M, N)$ as $R$-modules for all $R$-modules $M$ and $N$. Thus, $\operatorname{Ext}_{S}^{i}\left(K, H_{(I, x) S}^{j+1}(S)\right)=$ $\operatorname{Ext}_{R}^{i}\left(K, H_{I}^{j}(R)\right)$. The last statement then follows by induction on $n$.

Definition 3.2 ( $p$-independent, $p$-base). Let $K$ be a field of characteristic $p>0$. A finite set of elements $T_{1}, \ldots, T_{\ell} \in K \backslash K^{p}$ is called $p$-independent if $\left[K^{p}\left[T_{1}, \ldots, T_{\ell}\right]\right.$ : $\left.K^{p}\right]=p^{\ell}$. An infinite set of elements in $K \backslash K^{p}$ is $p$-independent if every finite subset is $p$-independent. A maximal $p$-independent subset of $K^{p} \backslash K$ is called a $p$-base for $K$.

Remark 3.3. We recall some results pertaining to the Cohen Structure Theorems that will be useful in proving that our new invariants are well defined; please see [5] for details.

For any field $K$ of characteristic $p>0$, there exists a complete (unramified) Noetherian discrete valuation ring (DVR) of mixed characteristic of the form $(V, p V, K)$. In fact, if $(V, p V, K)$ and $\left(W, p W, K^{\prime}\right)$ are complete Noetherian DVRs of mixed characteristic $p>0$ such that $K \cong K^{\prime}$, then $V \cong W$ as well. Indeed, given an isomorphism $\varphi: K \rightarrow K^{\prime}$, take a $p$-base $\Lambda$ for $K$, and let $\Lambda^{\prime}$ be the corresponding $p$-base for $K^{\prime}$ 
determined by $\varphi$. If $T \subseteq V$ is a lifting of $\Lambda$ to $V$ and $T^{\prime} \subseteq W$ is a lifting of $\Lambda^{\prime}$ to $W$, then the natural bijection $T \rightarrow T^{\prime}$ extends uniquely to an isomorphism $V \rightarrow W$. Every unramified complete regular local ring of mixed characteristic $p>0$ is isomorphic to $V \llbracket x_{1}, \ldots, x_{n} \rrbracket$, where $(V, p V, K)$ is a complete (unramified) Noetherian DVR of mixed characteristic $p$.

Suppose that $(R, m, K)$ is a complete local ring of mixed characteristic $p>0$. Then $R$ contains a subring $V^{\prime}$ that is called a coefficient ring for $R: V^{\prime}=V$ or $V^{\prime}=V / p^{\ell} V$ for some $\ell>1$, where $(V, p V, K)$ is a complete Noetherian DVR, and the induced map on residue fields, $V^{\prime} / p V^{\prime} \rightarrow R / m$, is an isomorphism. In fact, the Cohen Structure Theorems imply that given such a coefficient ring $V^{\prime}$ for $R$, there exists a surjection $\rho: V \llbracket x_{1}, \ldots, x_{n} \rrbracket \rightarrow R$ such that $\rho(V)=V^{\prime}$ (and we can take $n$ to be the embedding dimension of $R / p R$ ). A key point in the proof of this fact is that for every lifting $T$ of a $p$-base $\Lambda$ of $K$ to $R$, there is a unique coefficient ring $V^{\prime}$ for $R$ that contains $T$.

If $(R, m, K)$ is a complete local ring of equal characteristic $p>0$, then $R$ is a homomorphic image of some $K \llbracket x_{1}, \ldots, x_{n} \rrbracket$ by the Cohen Structure Theorems. Thus, if $(V, p V, K)$ is a complete Noetherian DVR, the composition $V \llbracket x_{1}, \ldots, x_{n} \rrbracket \rightarrow$ $K \llbracket x_{1}, \ldots, x_{n} \rrbracket \rightarrow R$ is surjective. Thus, any complete local ring $(R, m, K)$ such that $K$ has characteristic $p>0$ is the homomorphic image of $V \llbracket x_{1}, \ldots, x_{n} \rrbracket$, where $(V, p V, K)$ is a uniquely-determined (up to isomorphism) mixed characteristic complete Noetherian DVR.

Lemma 3.4. Suppose that $(R, m, K)$ is a complete local ring of mixed characteristic $p>0$, and let $V^{\prime}$ and $W^{\prime}$ be coefficient rings for $R$. Let $(V, p V, K)$ and $(W, p W, K)$ be complete Noetherian DVRs. Fix $n=\operatorname{dim}_{K}\left(m / m^{2}\right)$, and let $S_{1}=V \llbracket x_{1}, \ldots, x_{n} \rrbracket$ and $S_{2}=W \llbracket y_{1}, \ldots, y_{n} \rrbracket$. Then there exist surjections

$$
\rho_{1}: S_{1} \rightarrow R \quad \text { and } \quad \rho_{2}: S_{2} \rightarrow R
$$

and an isomorphism $\phi: S_{1} \rightarrow S_{2}$ such that $\rho_{1}(V)=V^{\prime}, \rho_{2}(W)=W^{\prime}$, and $\rho_{1}=\rho_{2} \circ \phi$.

Proof. Let $\Lambda$ and $\Delta$ be $p$-bases for $K$ corresponding to $V^{\prime}$ and $W^{\prime}$, respectively, and note that there are natural surjections $V \rightarrow V^{\prime}$ and $W \rightarrow W^{\prime}$. There exists a map $\rho_{1}: V \rightarrow V^{\prime}$ given by choosing preimages $t_{\lambda} \in V$ of $\lambda \in \Lambda$ under the composition $V \rightarrow V^{\prime} \rightarrow K$; similarly, take $\rho_{2}: W \rightarrow W^{\prime}$ given by preimages $s_{\delta} \in W$ of $\delta \in \Delta$ under the composition $W \rightarrow W^{\prime} \rightarrow K$. Pick elements $u_{1}, \ldots, u_{n} \in m$ such that the $\bar{u}_{i}$ form a basis for $m / m^{2}$, and extend $\rho_{1}$ to a map $S_{1} \rightarrow R$ by $\rho_{1}\left(x_{i}\right)=u_{i}$. Similarly, extend $\rho_{2}$ to a map $S_{2} \rightarrow R$ by $\rho_{1}\left(y_{i}\right)=u_{i}$. By construction, $\rho_{1}$ and $\rho_{2}$ are surjective.

For each $\lambda \in \Lambda$, take $\sigma_{\lambda} \in S_{2}$ be such that $\rho_{2}\left(\sigma_{\lambda}\right)=\rho_{1}\left(t_{\lambda}\right)$. Then there exists a unique coefficient ring $\widetilde{V}$ for $S_{2}$ such that $\sigma_{\lambda} \in \widetilde{V}$ for every $\lambda \in \Lambda$ (see Remark 3.3); moreover, $t_{\lambda} \mapsto \sigma_{\lambda}$ defines an isomorphism $\phi: V \rightarrow \widetilde{V}$. Extend this map to $\phi: S_{1} \rightarrow$ $S_{2}$ by $\phi\left(x_{i}\right)=y_{i}$. The induced map $K \cong S_{1} /\left(p, x_{1}, \ldots, x_{n}\right) \rightarrow S_{2} / \phi\left(p, x_{1}, \ldots, x_{n}\right) S_{2}$ is well defined, $\operatorname{char}\left(S_{2} / \phi\left(p, x_{1}, \ldots, x_{n}\right) S_{2}\right)=p$, and $p$ must be in the image of $\phi$. Thus, $\phi$ is surjective. As $S_{1}$ and $S_{2}$ have the same dimension, $\phi$ is, in fact, an isomorphism. Since $\left(\rho_{2} \circ \phi\right)\left(x_{j}\right)=\rho_{2}\left(y_{j}\right)=u_{j}=\rho_{1}\left(x_{j}\right)$ for each $1 \leq i \leq n$, and $\left(\rho_{2} \circ \phi\right)\left(t_{\lambda}\right)=$ $\rho_{2}\left(\sigma_{\lambda}\right)=\rho_{1}\left(t_{\lambda}\right)$ by construction, $\rho_{1}=\rho_{2} \circ \phi$.

We now prove that the Lyubeznik numbers in mixed characteristic are well defined. Our proof is inspired by Lyubeznik's in equal characteristic [15], but we employ the Cohen Structure Theorems in mixed characteristic, as we did in the proof of 
Lemma 3.4. The proof also relies on our extension of the study of the direct image functor $G$ (see Section 2.1) to rings that do not contain a field.

Theorem 3.5. Let $(R, m, K)$ be a local ring such that $K$ has characteristic $p>$ 0 , which admits a surjection $\pi: S \rightarrow R$, where $S$ is an $n$-dimensional unramified regular local ring of mixed characteristic. Let $I=\operatorname{Ker}(\pi)$, and take $i, j \geq 0$. Then $\operatorname{dim}_{K} \operatorname{Ext}_{S}^{i}\left(K, H_{I}^{n-j}(S)\right)$ is finite and depends only on $R, i$, and $j$.

Proof. Since each $\operatorname{dim}_{K} \operatorname{Ext}_{S}^{i}\left(K, H_{I}^{n-j}(S)\right)$ is finite (cf. [17, 20]), it remains to prove that these numbers are well defined. As the Bass numbers with respect to the maximal ideal are not affected by completion, we may assume that the rings are complete.

Fix a coefficient ring $W$ for $R$, and take $(V, p V, K)$ a complete Noetherian DVR such that $W=V$ or $W=V / p^{\ell} V$ for some $\ell>0$. First, take surjections $\pi: T \rightarrow R$ and $\pi^{\prime}: T^{\prime} \rightarrow R$, where $T=V \llbracket x_{1}, \ldots, x_{n-1} \rrbracket$ and $T^{\prime}=V \llbracket y_{1}, \ldots, y_{n^{\prime}-1} \rrbracket$ for some $n, n^{\prime} \geq 0$, such that $\pi(V)=W$, and $\left.\pi\right|_{V}=\left.\pi^{\prime}\right|_{V}$. Let $I=\operatorname{Ker}(\pi)$ and $I^{\prime}=\operatorname{Ker}\left(\pi^{\prime}\right)$.

Let $T^{\prime \prime}=V \llbracket x_{1}, \ldots, x_{n-1}, y_{1}, \ldots, y_{n^{\prime}-1} \rrbracket$, and let $\pi^{\prime \prime}: T^{\prime \prime} \rightarrow R$ be the surjection defined by $\left.\pi^{\prime \prime}\right|_{V}=\left.\pi\right|_{V}=\left.\pi^{\prime}\right|_{V}, \pi^{\prime \prime}\left(x_{j}\right)=\pi\left(x_{j}\right)$, and $\pi^{\prime \prime}\left(y_{j}\right)=\pi^{\prime}\left(y_{j}\right)$. Let $I^{\prime \prime}=$ $\operatorname{ker}\left(\pi^{\prime \prime}\right)$, and let $\alpha: T \hookrightarrow T^{\prime \prime}$ denote the natural injection, so that $\pi^{\prime \prime} \circ \alpha=\pi$.

As $\pi$ is surjective, there exist $f_{1}, \ldots, f_{n^{\prime}-1} \in T$ such that $\pi^{\prime \prime}\left(y_{j}\right)=\pi\left(f_{j}\right)$ for each $1 \leq j \leq n^{\prime}-1$. Then $y_{j}-f_{j} \in \operatorname{Ker}\left(\pi^{\prime \prime}\right)$. Note that $\beta: T^{\prime \prime} \rightarrow T$, defined by $\beta\left(x_{j}\right)=x_{j}, \beta\left(y_{j}\right)=f_{j}$, and $\left.\beta\right|_{V}=\operatorname{id}_{V}$, is a splitting of $\alpha$. Then $I^{\prime \prime}=\operatorname{Im}(\alpha) \oplus \operatorname{ker}(\beta)=$ $\left(I, y_{1}-f_{1}, \ldots, y_{n^{\prime}-1}-f_{n^{\prime}-1}\right) T^{\prime \prime}$. Since

$$
\nu, x_{1}, \ldots, x_{n-1}, y_{1}-f_{1}, \ldots, y_{n^{\prime}-1}-f_{n^{\prime}-1}
$$

form a regular system of parameters for $T^{\prime \prime}$, Lemma 3.1 implies that

$$
\operatorname{dim}_{K} \operatorname{Ext}_{T}^{i}\left(K, H_{I}^{n-j}(T)\right)=\operatorname{dim}_{K} \operatorname{Ext}_{T^{\prime \prime}}^{i}\left(K, H_{I^{\prime \prime}}^{n+n^{\prime}-j}\left(T^{\prime \prime}\right)\right) .
$$

By an analogous argument, we also have that

$$
\operatorname{dim}_{K} \operatorname{Ext}_{T^{\prime}}^{i}\left(K, H_{I^{\prime}}^{n^{\prime}-j}\left(T^{\prime}\right)\right)=\operatorname{dim}_{K} \operatorname{Ext}_{T^{\prime \prime}}^{i}\left(K, H_{I^{\prime \prime}}^{n+n^{\prime}-j}\left(T^{\prime \prime}\right)\right) .
$$

Now we proceed to the general case. Take complete Noetherian DVRs $(V, p V, K)$ and $(W, p W, K)$, let $S=V \llbracket x_{1}, \ldots, x_{n-1} \rrbracket$ and $S^{\prime}=W \llbracket y_{1}, \ldots, y_{n^{\prime}-1} \rrbracket$ for some $n, n^{\prime} \geq$ 0 , and take surjections $\pi: S \rightarrow R$ and $\pi^{\prime}: S^{\prime} \rightarrow R$, where $V^{\prime}$ and $W^{\prime}$ are coefficient rings for $R,\left.\pi\right|_{V}$ is a surjection of $V$ onto a $V^{\prime}$, and $\left.\pi\right|_{W}$ is a surjection of $W$ onto a $W^{\prime}$.

Let $N=\operatorname{dim}_{K}\left(m / m^{2}\right)+1$. Let $S_{1}=V \llbracket x_{1}, \ldots, x_{N} \rrbracket$ and $S_{2}=W \llbracket y_{1}, \ldots, y_{N} \rrbracket$, and let $\rho_{1}: S_{1} \rightarrow R$, and $\rho_{2}: S_{2} \rightarrow R$, and $\phi: S_{1} \rightarrow S_{2}$ be the maps ensured by Lemma 3.4; i.e., $\rho_{1}(V)=V^{\prime}$ and $\rho_{2}(W)=W^{\prime}$, and $\phi$ is an isomorphism, and $\rho_{1}=\rho_{2} \circ \phi$. By our initial case,

$$
\begin{aligned}
& \operatorname{dim}_{K} \operatorname{Ext}_{S_{2}}^{i}\left(K, H_{I}^{N-j}\left(S_{1}\right)\right)=\operatorname{dim}_{K} \operatorname{Ext}_{S}^{i}\left(K, H_{I}^{n-j}(S)\right) \text { and } \\
& \operatorname{dim}_{K} \operatorname{Ext}_{S_{2}}^{i}\left(K, H_{\phi(I)}^{N-j}\left(S_{2}\right)\right)=\operatorname{dim}_{K} \operatorname{Ext}_{S^{\prime}}^{i}\left(K, H_{I^{\prime}}^{n^{\prime}-j}\left(S^{\prime}\right)\right) .
\end{aligned}
$$

In addition, the isomorphism $\phi$ allows us to finally deduce that

$$
\operatorname{dim}_{K} \operatorname{Ext}_{S}^{i}\left(K, H_{I}^{N-j}\left(S_{1}\right)\right)=\operatorname{dim}_{K} \operatorname{Ext}_{S_{2}}^{i}\left(K, H_{\phi(I)}^{N-j}\left(S_{2}\right)\right) .
$$


Definition 3.6 (Lyubeznik numbers in mixed characteristic). Let $(R, m, K)$ be a local ring such that $K$ has characteristic $p>0$. By the Cohen Structure Theorems, the completion of $R$ at $m, \widehat{R}$, admits a surjection $\pi: S \rightarrow \widehat{R}$, where $S$ is an unramified regular local ring of mixed characteristic. Let $I=\operatorname{Ker}(\pi)$ and $n=\operatorname{dim}(S)$, and take $i, j \geq 0$. Then the Lyubeznik number in mixed characteristic of $R$ with respect to $i$ and $j$ is defined as

$$
\widetilde{\lambda}_{i, j}(R)=\operatorname{dim}_{K} \operatorname{Ext}_{S}^{i}\left(K, H_{I}^{n-j}(S)\right) .
$$

By Theorem 3.5, the $\widetilde{\lambda}_{i, j}(R)$ is well defined, finite, and depend only on $R, i$, and $j$.

Remark 3.7. In Definition 3.6, we need to complete $R$ for the Cohen Structure Theorems to ensure the existence of a surjection from an unramified regular local ring $S$ of mixed characteristic. If, without completing, a surjection from such an $S$ onto $R$ exists, and $I$ denotes its kernel, then $\widetilde{\lambda}_{i, j}(R)=\operatorname{dim}_{K} \operatorname{Ext}_{\widehat{S}}^{i}\left(K, H_{I \widehat{S}}^{n-j}(\widehat{S})\right)=$ $\operatorname{dim}_{K} \operatorname{Ext}_{S}^{i}\left(K, H_{I}^{n-j}(S)\right)$.

Remark 3.8. Fix a local ring $(R, m, K)$ of equal characteristic $p>0$. There exists a surjection from an $n$-dimensional unramified regular local ring of mixed characteristic, $\pi: S \rightarrow \widehat{R}$, and the induced map $\pi^{\prime}: S / p S \rightarrow \widehat{R}$ is also surjective. If $I=\operatorname{Ker}(\pi)$ and $I^{\prime}=\operatorname{Ker}\left(\pi^{\prime}\right)$, then $I$ is the preimage of $I^{\prime}$ under the canonical surjection $S \rightarrow S / p S$. In this case, both the Lyubeznik numbers of $R, \lambda_{i, j}(R)=$ $\operatorname{dim}_{K} \operatorname{Ext}_{S / p S}^{i}\left(K, H_{I^{\prime}}^{n-j-1}(S / p S)\right)$, and the Lyubeznik numbers in mixed characteristic of $R, \widetilde{\lambda}_{i, j}(R)=\operatorname{dim}_{K} \operatorname{Ext}_{S}^{i}\left(K, H_{I}^{n-j}(S)\right)$, are defined.

Remark 3.8 naturally incites the following question.

Question 3.9. For every local ring $(R, m, K)$ of equal characteristic $p>0$, and all $i, j \geq 0$, does $\widetilde{\lambda}_{i, j}(R)=\lambda_{i, j}(R)$ ?

In Corollary 5.3, we prove an affirmative answer to Question 3.9 when $R$ is CohenMacaulay, or when $\operatorname{dim}(R) \leq 2$. However, Remark 6.11 and Theorem 6.12 give an example of a Stanley-Reisner ring over $\mathbb{F}_{2}$ for which the answer is negative.

Remark 3.10. If $(S, m)$ is an unramified regular local ring of mixed characteristic $p>0, I$ is an ideal of $S$, and $i, j \geq 0$, then inj. $\operatorname{dim} H_{m}^{j} H_{I}^{i}(S) \leq 1$ and inj. $\operatorname{dim} H_{I}^{i}(S) \leq$ $\operatorname{dim} \operatorname{Supp} H_{I}^{i}(S)+1$ [29, Theorem 5.1].

We will use the following several times in our investigation of the Lyubeznik numbers in mixed characteristic.

Remark 3.11. Given a regular local $\operatorname{ring}(R, m, K)$ of dimension $d, H_{J}^{d}(R) \neq 0$ if and only if $\sqrt{J}=m$ by the Hartshorne-Lichtenbaum Vanishing Theorem [10, Theorem $3.1]$.

The Lyubeznik numbers in mixed characteristic satisfy some vanishing properties similar to those that hold for the Lyubeznik numbers.

Proposition 3.12. For a local ring $(R, m, K)$ of dimension d such that $K$ has characteristic $p>0$, the following hold.

(i) $\widetilde{\lambda}_{i, j}(R)=0$ if $j>d$ or $i>j+1$.

(ii) $\widetilde{\lambda}_{d, d}(R) \neq 0$. 
(iii) If $R$ is a complete intersection, then $\widetilde{\lambda}_{i, j}(R)=1$ if $i=j=d$, and $\widetilde{\lambda}_{i, j}(R)=0$ otherwise.

Proof. Replace $R$ by its completion at $m$, and fix a surjection $\pi: S \rightarrow R$, where $(S, \eta, K)$ is a complete unramified regular local ring of mixed characteristic and of dimension $n$. Let $I=\operatorname{Ker}(\pi)$.

For (i), the first statement holds since $H_{I}^{n-j}(S)=0$ for $j>\operatorname{dim}(S / I)=\operatorname{dim}(R)=$ $d$, and the second since by Remark 3.10, inj. $\operatorname{dim}_{S} H_{I}^{n-j}(S) \leq \operatorname{dim}_{S} H_{I}^{n-j}(S)+1 \leq$ $j+1$.

To prove (ii), first note that by an analogous argument to the proof of [15, Property 4.4(iii)], one can show that $H_{\eta}^{d} H_{I}^{n-d}(S) \neq 0$. We recall the proof's strategy: Using the Mayer-Vietoris sequence, we may reduce to the case that $R$ is equidimensional. The result then follows by applying Remark 3.11 and the spectral sequence $H_{\eta}^{p} H_{I}^{q}(S) \underset{p}{\Rightarrow}$ $H_{\eta}^{p+q}(S)$ simultaneously [9].

We will prove that $\widetilde{\lambda}_{d, d}(R) \neq 0$ by contradicting the fact that $H_{\eta}^{d} H_{I}^{n-d}(S) \neq$ 0 . Suppose that $\tilde{\lambda}_{d, d}(R)=\operatorname{dim}_{K} \operatorname{Ext}_{S}^{d}\left(K, H_{I}^{n-d}(S)\right)=0$. We claim that for every $S$-module $M$ of finite length, $\operatorname{Ext}_{S}^{d}\left(M, H_{I}^{n-d}(S)\right)=0$. We will prove this by induction on $h=\operatorname{length}_{S}(M)$. If $h=1$, then $M=K$, and the statement holds by assumption. Suppose that the statement is true for all $N$ with $\operatorname{length}_{S} N \leq h$, and take $M$ with length $_{S} M=h+1$. Then there exists a short exact sequence $0 \rightarrow K \rightarrow M \rightarrow M^{\prime} \rightarrow 0$, where $M^{\prime}$ is an $S$-module of length $h$. We obtain the following long exact sequence in Ext:

$$
\cdots \rightarrow \operatorname{Ext}_{S}^{d}\left(M^{\prime}, H_{I}^{n-d}(S)\right) \rightarrow \operatorname{Ext}_{S}^{d}\left(M, H_{I}^{n-d}(S)\right) \rightarrow \operatorname{Ext}_{S}^{d}\left(K, H_{I}^{n-d}(S)\right) \rightarrow \cdots .
$$

Now, $\operatorname{Ext}_{S}^{d}\left(K, H_{I}^{n-d}(S)\right)=\operatorname{Ext}_{S}^{d}\left(M^{\prime}, H_{I}^{n-d}(S)\right)=0$ by the inductive hypothesis, so that $\operatorname{Ext}_{S}^{d}\left(M, H_{I}^{n-d}(S)\right)=0$, and the claim follows. Therefore, for all $\ell \geq 1$, $\operatorname{Ext}_{S}^{d}\left(S / \eta^{\ell}, H_{I}^{n-d}(S)\right)=0$. Then $H_{\eta}^{d} H_{I}^{n-d}(S)=\underset{\vec{\ell}}{\lim _{\vec{Z}}} \operatorname{Ext}_{S}^{d}\left(S / \eta^{\ell}, H_{I}^{n-d}(S)\right)=0$, the sought contradiction.

To prove (iii), take $(V, p V, K)$ a complete Noetherian DVR, let $S=V \llbracket x_{1}, \ldots, x_{n} \rrbracket$, and consider a regular sequence $f_{1}, \ldots, f_{\ell} \in S$ such that $R \cong S /\left(f_{1}, \ldots, f_{\ell}\right)$.

If $\ell=1$, since $\operatorname{Ext}_{S}^{i}\left(K, S_{f_{1}}\right)=0$ for $i \geq 0$, the long exact sequence in Ext induced by the short exact sequence $0 \rightarrow S \rightarrow S_{f_{1}} \rightarrow H_{\left(f_{1}\right)}^{1}(S) \rightarrow 0$ implies that each $\operatorname{Ext}_{S}^{i}\left(K, H_{\left(f_{1}\right)}^{1}(S)\right) \cong \operatorname{Ext}_{S}^{i+1}(K, S)$; the vanishing is clear, and the statement follows.

Suppose that the formula holds for all such rings, such that the regular sequence has length $\ell$, for some $\ell \geq 1$. The long exact sequence in Ext induced by the short exact sequence

$$
0 \rightarrow H_{\left(f_{1}, \ldots, f_{\ell}\right)}^{\ell}(S) \rightarrow H_{\left(f_{1}, \ldots, f_{\ell}\right)}^{\ell}\left(S_{f_{\ell+1}}\right) \rightarrow H_{\left(f_{1}, \ldots, f_{\ell+1}\right)}^{\ell+1}(S) \rightarrow 0
$$

indicates that each $\operatorname{Ext}_{S}^{i}\left(K, H_{\left(f_{1}, \ldots, f_{\ell+1}\right)}^{\ell+1}(S)\right) \cong \operatorname{Ext}_{S}^{i+1}\left(K, H_{\left(f_{1}, \ldots, f_{\ell}\right)}^{\ell}(S)\right)$, and the result follows.

\section{Existence of the highest Lyubeznik number in mixed characteristic}

The aim of this section is to prove that the Lyubeznik numbers in mixed characteristic vanish if either index exceeds the ring's dimension. A key step will be Theorem 4.9 
on the injective dimension of certain local cohomology modules of unramified regular local rings of mixed characteristic. We begin this section by investigating some properties of injective modules in the mixed characteristic setting.

Lemma 4.1. Let $(V, p V, K)$ be a complete (unramified) Noetherian DVR of mixed characteristic $p>0$, and let $S=V \llbracket x_{1} \ldots, x_{n} \rrbracket$. Let $M$ be an $S$-module such that $\operatorname{dim}_{K} \operatorname{Ext}_{S}^{i}(K, M)$ is finite for all $i \geq 0$, and such that multiplication by $p$ on $M$ is surjective. Then for all $i \geq 0$,

$$
\operatorname{dim}_{K} \operatorname{Ext}_{S}^{i}(K, M)=\operatorname{dim}_{K} \operatorname{Ext}_{S / p S}^{i}\left(K, \operatorname{Ann}_{M}(p S)\right) .
$$

Proof. Let $R$ denote $S / p S$, and let $N=\operatorname{Ann}_{M}(p S)$. Then the short exact sequence $0 \rightarrow N \rightarrow M \stackrel{\cdot p}{\rightarrow} M \rightarrow 0$ induces the long exact sequence

$$
\cdots \rightarrow \operatorname{Ext}_{S}^{i-1}(K, M) \rightarrow \operatorname{Ext}_{S}^{i}(K, N) \rightarrow \operatorname{Ext}_{S}^{i}(K, M) \stackrel{\cdot p}{\rightarrow} \operatorname{Ext}_{S}^{i}(K, M) \rightarrow \cdots .
$$

Since multiplication by $p$ is zero on $\operatorname{Ext}_{S}^{j}(K, M)$ for all $j \geq 0$, we have short exact sequences

$$
0 \rightarrow \operatorname{Ext}_{S}^{i-1}(K, M) \rightarrow \operatorname{Ext}_{S}^{i}(K, N) \rightarrow \operatorname{Ext}_{S}^{i}(K, M) \rightarrow 0
$$

for all $i \geq 0$, so that

$$
\operatorname{dim}_{K} \operatorname{Ext}_{S}^{i}(K, N)=\operatorname{dim}_{K} \operatorname{Ext}_{S}^{i-1}(K, M)+\operatorname{dim}_{K} \operatorname{Ext}_{S}^{i}(K, M) .
$$

Let $\boldsymbol{x}$ denote the sequence $x_{1}, \ldots, x_{n}$ in $S$. The $\operatorname{Koszul}$ complex $\mathcal{K}_{\bullet}(p, \boldsymbol{x} ; N)$ satisfies $\mathcal{K}_{\bullet}(p, \boldsymbol{x} ; N)=\mathcal{K}_{\bullet}(p ; N) \otimes_{S} \mathcal{K}_{\bullet}(\boldsymbol{x} ; N)$, so that

$$
\begin{aligned}
\mathcal{K}_{i}(p, \boldsymbol{x} ; N) & =\left(\mathcal{K}_{0}(p ; N) \otimes_{S} \mathcal{K}_{i}(\boldsymbol{x} ; N)\right) \oplus\left(\mathcal{K}_{1}(p ; N) \otimes_{S} \mathcal{K}_{i-1}(\boldsymbol{x} ; N)\right) \\
& \cong \mathcal{K}_{i}(\boldsymbol{x} ; N) \oplus \mathcal{K}_{i-1}(\boldsymbol{x} ; N) .
\end{aligned}
$$

Moreover, since $N$ is killed by $p$, the differentials are given, up to a sign, by the differentials on the $\mathcal{K}_{\bullet}(\boldsymbol{x} ; N)$. This complex is naturally a complex over $R$ after replacing $x_{1}, \ldots, x_{n}$ by their images in $R$. We can compute $\operatorname{Ext}_{R}^{i}(K, N)$ using this complex. On the other hand, we can compute $\operatorname{Ext}_{S}^{i}(K, N)$ using the complex $\mathcal{K}_{\bullet}(p, \boldsymbol{x} ; N)$. Thus,

$$
\operatorname{dim}_{K} \operatorname{Ext}_{R}^{i-1}(K, N)+\operatorname{dim}_{K} \operatorname{Ext}_{R}^{i}(K, N)=\operatorname{dim}_{K} \operatorname{Ext}_{S}^{i}(K, N),
$$

so that, noting (4.1.1),

$$
\begin{aligned}
& \operatorname{dim}_{K} \operatorname{Ext}_{S}^{i-1}(K, M)+\operatorname{dim}_{K} \operatorname{Ext}_{S}^{i}(K, M) \\
& \quad=\operatorname{dim}_{K} \operatorname{Ext}_{R}^{i-1}(K, N)+\operatorname{dim}_{K} \operatorname{Ext}_{R}^{i}(K, N) .
\end{aligned}
$$

The statement now follows by an induction argument on $i \geq 0$ : The basis case follows by taking $i=0$ in (4.1.2). Assuming the statement holds for $i-1$, the conclusion also follows by (4.1.2).

Lemma 4.2. Let $(V, p V, K)$ be a complete (unramified) Noetherian DVR of mixed characteristic $p>0$, and let $S=V \llbracket x_{1} \ldots, x_{n} \rrbracket$. If $m$ denotes the maximal ideal of $S$, let $M$ be a $D(S, V)$-module supported only at $m$ such that $\operatorname{dim}_{K} \operatorname{Ext}_{S}^{i}(K, M)$ is finite for every $i \geq 0$. Then $M$ is injective if and only if multiplication by $p$ on $M$ is surjective. 
Proof. If $M$ is injective, then it is isomorphic to a finite direct sum of copies of $E_{S}(K)$. By applying Matlis duality to the exact sequence $0 \rightarrow S \stackrel{\cdot p}{\rightarrow} S \rightarrow S / p S \rightarrow 0$, we conclude that multiplication by $p$ is surjective on $E_{S}(K)$, and so, on $M$.

Assume that multiplication by $p$ on $M$ is surjective. Since $N$ is a $D(S / p S, K)$ module supported only at the maximal ideal $\left(x_{1}, \ldots, x_{n}\right)$ of $S / p S, N$ is an injective $S / p S$-module by [18, Lemma], and so, by Lemma 4.1 ,

$$
\operatorname{dim}_{K} \operatorname{Ext}_{S}^{i}(K, M)=\operatorname{dim}_{K} \operatorname{Ext}_{S / p S}^{i}(K, N)=0
$$

for every $i>0$. Since $M$ is supported only at $m$, the only nonzero Bass number of $M$ is $\operatorname{dim}_{K} \operatorname{Hom}_{S}(K, M)$, and $M$ is injective.

Our next goal is to show, in Theorem 4.9, that for an unramified regular local ring $S$ of mixed characteristic and dimension $n$, if $I$ is an ideal of $S$ such that $S / I$ has dimension $d$, then inj. $\operatorname{dim}_{S} H_{I}^{n-d}(S)=d$. We prove this statement by induction on $d$; the following lemmas are devoted to proving the base cases, $d=1$ and $d=2$.

Lemma 4.3. Let $(V, p V, K)$ be a complete (unramified) Noetherian DVR of mixed characteristic $p>0$, and let $S=V \llbracket x_{1} \ldots, x_{n} \rrbracket$. Let $m$ denote the maximal ideal of $S$, and let $I$ be an ideal of $S$ such that $\operatorname{dim}(S / I)=1$. Then $H_{m}^{0} H_{I}^{n}(S)=0$ and $H_{m}^{1} H_{I}^{n}(S) \cong E_{S}(K)$.

Proof. Let $f \in m$ be an element not in any minimal prime of $I$, so that $\sqrt{I+f S}=m$. We have the short exact sequence $0 \rightarrow H_{I}^{n}(S) \rightarrow H_{I}^{n}\left(S_{f}\right) \rightarrow H_{I+f S}^{n+1}(S) \cong E_{S}(K) \rightarrow 0$. Since $f \in m$ and $H_{I}^{n}\left(S_{f}\right) \cong H_{I}^{n}(S)_{f}, H_{m}^{0} H_{I}^{n}\left(S_{f}\right)=0$, which implies that $H_{m}^{0} H_{I}^{n}(S)=$ 0 . Moreover, $H_{m}^{1} H_{I}^{n}(S) \cong E_{S}(K)$.

Lemma 4.4. Let $(V, p V, K)$ be a complete (unramified) Noetherian DVR of mixed characteristic $p>0$, and let $S=V \llbracket x_{1} \ldots, x_{n} \rrbracket$. Let $I$ be an ideal of $S$ of pure dimension two. Then $H_{I}^{n}(S)$ is an injective $S$-module supported only at the maximal ideal of $S$.

Proof. Let $R$ denote $S / p S$. The short exact sequence $0 \rightarrow S \stackrel{\cdot p}{\rightarrow} S \rightarrow R \rightarrow 0$ induces the long exact sequence $\cdots \rightarrow H_{I}^{n}(S) \stackrel{\cdot p}{\rightarrow} H_{I}^{n}(S) \rightarrow H_{I}^{n}(R) \rightarrow 0$, where $H_{I}^{n}(R)=0$ by Remark 3.11, as $\sqrt{I+p S}$ cannot be the maximal ideal. Thus, multiplication by $p$ on $H_{I}^{n}(S)$ is surjective.

For every prime ideal $P$ of $S$ not containing $I, H_{I S_{P}}^{n}\left(S_{P}\right)=0$ : Indeed, if $I \subseteq$ $P$ and $\operatorname{dim}(S / P)=2$, then $H_{I S_{P}}^{n}\left(S_{P}\right)=0$ because $\operatorname{dim}\left(S_{P}\right)<n$. If $I \subseteq P$ and $\operatorname{dim}(S / P)=2$, then $H_{I S_{P}}^{n}\left(S_{P}\right)=0$ by Remark 3.11 because $I$ has pure dimension 2 and $\sqrt{I S_{P}} \neq P S_{P}$.

Therefore, $H_{I}^{n}(S)$ is a $D(S, V)$-module supported only at the maximal ideal. Since $\operatorname{dim}_{K} \operatorname{Hom}_{S}\left(K, H_{I}^{n}(S)\right)$ is finite, $H_{I}^{n}(S)$ is injective by Lemma 4.2.

Lemma 4.5. Let $(V, p V, K)$ be a complete (unramified) Noetherian DVR of mixed characteristic $p>0$, let $S=V \llbracket x_{1} \ldots, x_{n} \rrbracket$, and let $m$ denote its maximal ideal. For an ideal $I$ of $S$ of pure dimension two, $H_{m}^{0} H_{I}^{n-1}(S)=H_{m}^{1} H_{I}^{n-1}(S)=0$. Moreover, $H_{I}^{n}(S) \cong \bigoplus_{i=1}^{\alpha} E_{S}(K)$ for some $\alpha \geq 0$, and $H_{m}^{2} H_{I}^{n-1}(S) \cong \bigoplus_{i=1}^{\alpha+1} E_{S}(K)$. In particular, $H_{m}^{2} H_{I}^{n-1}(S)$ is an injective $S$-module. 
Proof. Take $f \in m$ not in any minimal prime of $I$, so that $\sqrt{I+f S} \subsetneq m$. Applying Remark 3.11, since $H_{I}^{n}(S)$ is supported at $m$ by Lemma 4.4, we obtain the exact sequence

$$
0 \rightarrow H_{I}^{n-1}(S) \rightarrow H_{I}^{n-1}\left(S_{f}\right) \rightarrow H_{I+f S}^{n}(S) \rightarrow H_{I}^{n}(S) \rightarrow 0 .
$$

If $M$ denotes the kernel of the last nonzero map, splitting the sequence into two short exact sequences, we obtain

$$
0 \rightarrow H_{I}^{n-1}(S) \rightarrow H_{I}^{n-1}\left(S_{f}\right) \rightarrow M \rightarrow 0 \quad \text { and } \quad 0 \rightarrow M \rightarrow H_{I+f S}^{n}(S) \rightarrow H_{I}^{n}(S) \rightarrow 0 .
$$

These induce the long exact sequences

$$
\begin{aligned}
& 0 \longrightarrow H_{m}^{0} H_{I}^{n-1}(S) \longrightarrow H_{m}^{0} H_{I}^{n-1}\left(S_{f}\right) \longrightarrow H_{m}^{0}(M) \\
& \longrightarrow H_{m}^{1} H_{I}^{n-1}(S) \longrightarrow H_{m}^{1} H_{I}^{n-1}\left(S_{f}\right) \longrightarrow H_{m}^{1}(M) \longrightarrow \\
& \longrightarrow H_{m}^{2} H_{I}^{n-1}(S) \longrightarrow H_{m}^{2} H_{I}^{n-1}\left(S_{f}\right) \longrightarrow H_{m}^{2}(M) \longrightarrow 0, \quad \text { and } \\
& \left.\longrightarrow H_{m}^{0}(M) \longrightarrow H_{m}^{0} H_{I+f S}^{n}(S) \longrightarrow H_{m}^{0} H_{I}^{n}(S)\right] \\
& \longrightarrow H_{m}^{1}(M) \longrightarrow H_{m}^{1} H_{I+f S}^{n}(S) \longrightarrow H_{m}^{1} H_{I}^{n}(S) \\
& \longrightarrow H_{m}^{2}(M) \longrightarrow H_{m}^{2} H_{I+f S}^{n}(S) \longrightarrow H_{m}^{2} H_{I}^{n}(S) \longrightarrow 0 .
\end{aligned}
$$

Since for all $j \geq 0, H_{m}^{j} H_{I}^{n-1}\left(S_{f}\right)=0$, we know that $H_{m}^{0} H_{I}^{n-1}(S)=H_{m}^{2}(M)=0$, $H_{m}^{0}(M) \cong H_{m}^{1} H_{I}^{n-1}(S)$, and $H_{m}^{1}(M) \cong H_{m}^{2} H_{I}^{n-1}(S)$. Since $\operatorname{dim}(S /(I+f S))=1$, $H_{m}^{0} H_{I+f S}^{n}(S)=0$ by Lemma 4.3 , which implies that $H_{m}^{1} H_{I}^{n-1}(S)=H_{m}^{0}(M)=0$. In addition, $H_{m}^{1} H_{I}^{n}(S)=H_{m}^{2} H_{I}^{n}(S)=0$ by Lemma 4.4 . Thus, we have a short exact sequence

$$
0 \rightarrow H_{m}^{0} H_{I}^{n}(S) \rightarrow H_{m}^{2} H_{I}^{n-1}(S) \rightarrow H_{m}^{1} H_{I+f S}^{n}(S) \rightarrow 0
$$

By Lemma 4.4, $H_{I}^{n}(S)$ is an injective $S$-module supported only at $m$, and its Bass numbers are finite by $[17,20]$, so $H_{m}^{0} H_{I}^{n}(S)=H_{I}^{n}(S) \cong \bigoplus_{i=1}^{\alpha} E_{S}(K)$ for some $\alpha \geq 0$. Moreover, by Lemma 4.3, $H_{m}^{1} H_{I+f S}^{n}(S) \cong E_{S}(K)$. Therefore, the short exact sequence $0 \rightarrow \bigoplus_{i=1}^{\alpha} E_{S}(K) \rightarrow H_{m}^{2} H_{I}^{n-1}(S) \rightarrow E_{S}(K) \rightarrow 0$ splits, so that $H_{m}^{2} H_{I}^{n-1}(S) \cong \bigoplus_{i=1}^{\alpha+1} E_{S}(K)$.

Corollary 4.6. Let $(V, p V, K)$ be a complete (unramified) Noetherian DVR of mixed characteristic $p>0$, and let $S=V \llbracket x_{1} \ldots, x_{n} \rrbracket$. Let $I$ be an ideal of $S$ of pure dimension two. Then for all $i, j \geq 0$ and every prime ideal $Q$ of $S, H_{Q}^{i} H_{I}^{j}\left(S_{Q}\right)$ is injective. 
Proof. This follows from Lemmas 4.3 and 4.5 .

Lemma 4.7. Let $(V, p V, K)$ be a complete (unramified) Noetherian DVR of mixed characteristic $p>0$, let $S=V \llbracket x_{1} \ldots, x_{n} \rrbracket$, and let $m$ denote its maximal ideal. Let $I$ be an ideal of $S$ such that $\operatorname{dim}(S / I)=2$. Then $H_{m}^{0} H_{I}^{n-1}(S)=H_{m}^{1} H_{I}^{n-1}(S)=0$ and $H_{m}^{2} H_{I}^{n-1}(S)$ is an injective $S$-module.

Proof. Take $J_{1}$ and $J_{2}$, ideals of pure dimensions one and two, respectively, such that $I=J_{1} \cap J_{2}$. By the Mayer-Vietoris sequence of local cohomology, $H_{I}^{n-1}(S)=$ $H_{J_{2}}^{n-1}(S)$. Thus, for all $j \geq 0, H_{m}^{j} H_{I}^{n-1}(S)=H_{m}^{j} H_{J_{2}}^{n-1}(S)$, and the result follows by Lemma 4.5.

The following proposition will allow us to use an induction argument in the proof of Theorem 4.9.

Proposition 4.8. Let $(V, p V, K)$ be a complete (unramified) Noetherian DVR of mixed characteristic $p>0$, let $S=V \llbracket x_{1} \ldots, x_{n} \rrbracket$, and let $m$ denote the maximal ideal of $S$. For an ideal $I$ of $S$ with $\operatorname{dim}(S / I)=d, H_{m}^{d} H_{I}^{n-d+1}(S)$ is an injective $S$-module and $H_{m}^{j} H_{I}^{n-d+1}(S)=0$ for $j>d$.

Proof. We proceed by induction on $d$. If $d=0$, the statement is clear, and for $d=$ 1 and $d=2$, the result holds by Lemmas 4.3 and 4.7 , respectively. Suppose that $d \geq 3$ and the statement holds for $d-1$. We fix an element $r \in m$ as follows: if $\operatorname{Ass}_{S} H_{I}^{n-d}(S)=\{m\}$, fix $r \in m$ not in any minimal prime of $I$; otherwise, fix $r \in m$ that is not in any minimal prime of $I$, nor in any minimal prime of $H_{I}^{n-d}(S)$, which is possible because $\operatorname{Ass}_{S} H_{I}^{n-d}(S)$ is finite (cf. [17, 20]). Then $H_{m}^{d} H_{I}^{n-d+1}(S) \cong$ $H_{m}^{d-1} H_{I+r S}^{n-d+2}(S)$ and $H_{m}^{j} H_{I}^{n-d+1}(S)=H_{m}^{j-1} H_{I+r S}^{n-d+2}(S)=0$ for $j>d$ as in the proof of [28, Proposition 2.1] because the conclusions of [28, Lemmas 2.3 and 2.4] hold in our case. Hence, the result follows.

Theorem 4.9. Let $(S, m, K)$ be either an unramified regular local ring of mixed characteristic, or a regular local ring containing a field. Let $n=\operatorname{dim}(S)$, and let $I$ be an ideal of $S$ such that $\operatorname{dim}(S / I)=d$. Then inj. $\operatorname{dim} H_{I}^{n-d}(S)=d$.

Proof. Since the theorem is already true for regular local rings that contain a field (cf. $[12,15,18]$ ), we will focus on the case where $S$ is an unramified regular local ring of mixed characteristic.

Our goal is to show that for every $j>d$ and every prime ideal $Q$ of $S$,

$$
\operatorname{Ext}_{S_{Q}}^{j}\left(S_{Q} / Q S_{Q}, H_{I S_{Q}}^{n-d}\left(S_{Q}\right)\right)=0
$$

by the relation of the Bass numbers with minimal injective resolutions. We may assume that $Q=m$ because if $Q \subsetneq m$, then $\operatorname{dim}\left(S_{Q} / I S_{Q}\right) \leq d-1$, and by Remark 3.10, inj. $\operatorname{dim}_{S_{Q}} H_{I S_{Q}}^{n-d}\left(S_{Q}\right) \leq \operatorname{dim}_{S_{Q}} H_{I S_{Q}}^{n-d}\left(S_{Q}\right)+1 \leq d$.

We proceed by induction on $n$. If $n=0$, then $S$ is a field and the result follows. Assume that for all such $S$ of dimension less than $n$, and all $d \leq n$, if $I$ is an ideal of $S$ such that $\operatorname{dim}(S / I)=d$, then (4.9.1) holds.

Let $E^{\bullet}=\left(E^{0} \rightarrow E^{1} \rightarrow E^{2} \rightarrow \ldots\right)$ be a minimal injective resolution of $H_{I}^{n-d}(S)$. By Remark 3.10, $E^{j}=0$ for $j>d+1$. For every prime ideal $Q$ of $S, S_{Q}$ is either an unramified regular local ring of mixed characteristic or a regular local ring containing a field. Moreover, $\operatorname{dim}\left(S_{Q} / I S_{Q}\right) \leq d-1$ for every prime ideal $Q \subsetneq m$. Thus, 
$\left(E^{d}\right)_{Q}=\left(E^{d+1}\right)_{Q}=0$ by the inductive hypothesis. Hence, $E^{d}$ and $E^{d+1}$ are supported only at $m$.

Let $M=\operatorname{Im}\left(E^{d-1} \rightarrow E^{d}\right)=\operatorname{Ker}\left(E^{d} \rightarrow E^{d+1}\right)$. Now, $H_{m}^{j} H_{I}^{n-d}(S)$ can be computed from the complex $H_{m}^{0}\left(E^{\bullet}\right)=\left(H_{m}^{0}\left(E^{0}\right) \rightarrow H_{m}^{0}\left(E^{1}\right) \rightarrow H_{m}^{0}\left(E^{2}\right) \rightarrow \ldots\right)$. Let $B^{j}=\operatorname{Im}\left(H_{m}^{0}\left(E^{j-1}\right) \rightarrow H_{m}^{0}\left(E^{j}\right)\right)$ and $Z^{j}=\operatorname{Ker}\left(H_{m}^{0}\left(E^{j}\right) \rightarrow H_{m}^{0}\left(E^{j+1}\right)\right)$. Note that $Z^{d}=M$ since $E_{d}$ and $E_{d+1}$ are supported only at $m$. Since $\operatorname{inj}$. $\operatorname{dim} Z^{j} \leq 1$ and $\operatorname{inj.} \operatorname{dim} H_{m}^{j} H_{I}^{n-d}(S) \leq 1$ by Remark 3.10, as in its proof in [29, Theorem 5.1], we obtain that $B^{j}$ is injective from the short exact sequences

$$
0 \rightarrow Z^{j} \rightarrow H_{m}^{0}\left(E^{j}\right) \rightarrow B^{j} \rightarrow 0 \text { and } 0 \rightarrow B^{j-1} \rightarrow Z^{j} \rightarrow H_{m}^{j} H_{I}^{n-d}(S) \rightarrow 0 .
$$

Since $H_{m}^{d} H_{I}^{n-d}(S)$ injective by Proposition 4.8, we know that $M=Z^{d}$ is injective by the second exact sequence in (4.9.2) with $j=d$. Therefore, $E_{d+1}=0$, so inj. $\operatorname{dim} H_{I}^{n-d}(S)=d$.

Theorem 4.9 and Proposition 3.12(i) imply that for a local ring $R$ with prime characteristic residue field, $\lambda_{i, j}(R)=0$ for $i>d$ or $j>d$, justifying the following nomenclature.

Definition 4.10 (Highest Lyubeznik number in mixed characteristic). For a local ring $(R, m, K)$ of dimension $d$ such that $K$ has characteristic $p>0$, the highest Lyubeznik number of $R$ in mixed characteristic is $\widetilde{\lambda}_{d, d}(R)$.

Note that the highest Lyubeznik number in mixed characteristic is nonzero by Proposition 3.12(ii).

We can also create a table of all Lyubeznik numbers in mixed characteristic, analogous to the Lyubeznik table defined for local rings containing a field.

Definition 4.11 (Lyubeznik table in mixed characteristic). For a local ring $(R, m, K)$ such that $K$ has characteristic $p>0$ and $d=\operatorname{dim}(R)$, the Lyubeznik table of $R$ in mixed characteristic is the $(d+1) \times(d+1)$ matrix $\widetilde{\Lambda}(R)$ defined by $\widetilde{\Lambda}(R)_{i, j}=\widetilde{\lambda}_{i, j}(R)$ for $0 \leq i, j \leq d$.

Remark 4.12. Recall that for a local ring $R$ of dimension $d$ containing a field, the Lyubeznik table of $R$ is defined as the $(d+1) \times(d+1)$ matrix $\Lambda(R)$ such that $\Lambda(R)_{i, j}=\lambda_{i, j}(R)$ for $0 \leq i, j \leq d$. This matrix contains all nonzero Lyubeznik numbers, and is also upper triangular, since $\lambda_{i, j}(R)=0$ if either $i>j$ or $j>d[15$, Properties 4.4]. On the other hand, Proposition 3.12 and Theorem 4.9 imply that the Lyubeznik table in mixed characteristic contains all nonzero Lyubeznik numbers in mixed characteristic. However, Proposition 3.12 only implies that the Lyubeznik table in mixed characteristic is zero below the subdiagonal; it is not known whether all entries below the diagonal must vanish. This is related to a question of Lyubeznik, which asks, assuming that $(S, m)$ is a regular local ring and $I$ is an ideal of $S$, whether local cohomology modules of the form $H_{m}^{i} H_{I}^{j}(S)$ must be injective [15, Question 3.8].

Question 4.13. What topological properties do the Lyubeznik numbers in mixed characteristic capture? In particular, what information is encoded in the highest Lyubeznik number? 


\section{Examples for which the Lyubeznik numbers and the Lyubeznik numbers in mixed characteristic agree}

In this section, we show that for certain local rings of equal characteristic $p>0$, the Lyubeznik numbers in mixed characteristic agree with the Lyubeznik numbers. First we deal with the case of Cohen-Macaulay rings, and then rings of small dimension.

Proposition 5.1. Let $(V, p V, K)$ be a complete (unramified) Noetherian DVR of mixed characteristic $p>0$, and let $S=V \llbracket x_{1} \ldots, x_{n} \rrbracket$. Let $I$ be an ideal of $S$ such that $S / I$ is a Cohen-Macaulay ring of equal characteristic $p$. Then for all $i, j \geq 0$,

$$
\operatorname{dim}_{K} \operatorname{Ext}_{S / p S}^{i}\left(K, H_{I S / p S}^{n-j}(S / p S)\right)=\operatorname{dim}_{K} \operatorname{Ext}_{S}^{i}\left(K, H_{I}^{n+1-j}(S)\right) .
$$

Proof. Let $R=S / p S$, so that the short exact sequence $0 \rightarrow S \stackrel{\cdot p}{\rightarrow} S \rightarrow R \rightarrow 0$ induces the long exact sequence

$$
\cdots \rightarrow H_{I}^{n-j}(R) \rightarrow H_{I}^{n+1-j}(S) \stackrel{\cdot p}{\rightarrow} H_{I}^{n+1-j}(S) \rightarrow \cdots .
$$

Let $d=\operatorname{dim}(S / I)$. Now, $H_{I}^{n-j}(R) \neq 0$ if and only if $j=d$ by [22, Theorem III.4.1], so that multiplication by $p$ on $H_{I}^{n+1-j}(S)$ is injective except when $j=d$. Moreover, since $S / I$ is Cohen-Macaulay, $H_{I}^{n-d}(S)=0$, so that multiplication by $p$ on every $H_{I}^{n+1-j}(S)$ is surjective. The result then holds by Lemma 4.1 .

The following result implies that the Lyubeznik numbers in mixed characteristic coincide with the original invariants for local rings of equal characteristic $p>0$ with separably closed residue field and dimension at most two.

Proposition 5.2. Let $(V, p V, K)$ be a complete (unramified) Noetherian DVR of mixed characteristic $p>0$ such that $K$ is separably closed. If $S=V \llbracket x_{1}, \ldots, x_{n} \rrbracket$ and $I$ is an ideal of $S$ containing $p$ such that $d:=\operatorname{dim}(S / I) \leq 2$, then

$$
\operatorname{dim}_{K} \operatorname{Ext}_{S / p S}^{d}\left(K, H_{I S / p S}^{n-d}(S / p S)\right)=\operatorname{dim}_{K} \operatorname{Ext}_{S}^{d}\left(K, H_{I}^{n-d+1}(S)\right) .
$$

Proof. Let $R=S / p S$, and first assume that $\operatorname{dim}(S / I)=0$. We have that $H_{I}^{n+1}(S) \cong$ $E_{S}(K)$ and $H_{I S / p S}^{n}(S / p S) \cong E_{S / p S}(K)$. In this case,

$$
\operatorname{dim}_{K} \operatorname{Hom}_{S / p S}\left(K, H_{I S / p S}^{n}(S / p S)\right)=1=\operatorname{dim}_{K} \operatorname{Hom}_{S}\left(K, H_{I}^{n+1}(S)\right) .
$$

Now assume that $\operatorname{dim}(S / I)=1$, so that the short exact sequence

$$
0 \rightarrow S \stackrel{\cdot p}{\rightarrow} S \rightarrow R \rightarrow 0
$$

induces the exact sequence $0 \rightarrow H_{I}^{n-1}(R) \rightarrow H_{I}^{n}(S) \stackrel{\cdot p}{\rightarrow} H_{I}^{n}(S) \rightarrow 0$, noting Remark 3.11. The proposition then follows in this case from Lemma 4.1.

Now assume that $\operatorname{dim}(S / I)=2$. First consider $I$ of pure dimension two. Let $\alpha$ be the number of connected components of $\operatorname{Spec}(A) \backslash\{m\}$, where $A=R / I$. In fact, $\alpha=\operatorname{dim}_{K} \operatorname{Ext}_{R}^{2}\left(K, H_{I}^{n-2}(S / p S)\right)$ (cf. [25, Proposition 2.2]).

We prove the statement by induction on $\alpha$. If $\alpha=1$, the short exact sequence (5.2.1) induces the short exact sequence $0 \rightarrow H_{I}^{n-2}(R) \rightarrow H_{I}^{n-1}(S) \stackrel{\cdot p}{\rightarrow} H_{I}^{n-1}(S) \rightarrow 0$, since $H_{I}^{n-1}(R)=0$ by [11, Theorem 2.9]. The statement then follows from Lemma 4.1. If $\alpha>1$, we pick ideals $J_{1}, \ldots, J_{\alpha}$ such that $I=J_{1} \cap \cdots \cap J_{\alpha}$, and each $J_{k}$ defines a 
connected component of $\operatorname{Spec}(\widehat{A}) \backslash\{m\}$. Let $J$ denote $J_{1} \cap \cdots \cap J_{\alpha-1}$. As $\sqrt{J+J_{\alpha}}=m$, $H_{J}^{n-1}(S) \oplus H_{J_{\alpha}}^{n-1}(S) \cong H_{I}^{n-1}(S)$ by the Mayer-Vietoris sequence, so that

$$
\operatorname{dim}_{K} \operatorname{Ext}_{S}^{2}\left(K, H_{I}^{n-1}(S)\right)=\operatorname{dim}_{K} \operatorname{Ext}_{S}^{2}\left(K, H_{J}^{n-1}(S)\right)+\operatorname{dim}_{K} \operatorname{Ext}_{S}^{2}\left(K, H_{J_{\alpha}}^{n-1}(S)\right),
$$

which equals $\alpha$. By Lemma 4.5, [15, Lemma 1.4], and [25, Proposition 2.2], the other numbers are determined by $\alpha$.

For the general case with $\operatorname{dim}(S / I)=2$, let $P_{1}, \ldots, P_{r}$ and $Q_{1}, \ldots, Q_{s}$ denote the minimal primes of $I$ of dimensions one and two, respectively. Let $J_{1}=P_{1} \cap \ldots \cap P_{r}$ and $J_{2}=Q_{1} \cap \ldots \cap Q_{s}$. We claim that $\operatorname{Ext}_{S}^{j}\left(K, H_{I}^{n-1}(S)\right) \cong \operatorname{Ext}_{S}^{j}\left(K, H_{J_{2}}^{n-1}(S)\right)$. Let $f_{1}, \ldots, f_{\ell} \in J_{2} \backslash I$ such that $I+\left(f_{1}, \ldots, f_{\ell}\right) S=J_{2}$. We proceed by induction on $\ell$; first assume that $\ell=1$. Since $H_{I}^{n-1}(S)=H_{J_{2}}^{n-1}(S), H_{I}^{n-1}\left(S_{f_{1}}\right)=0$. The exact sequence

$$
0 \rightarrow H_{I+f_{1} S}^{n-1}(S) \rightarrow H_{I}^{n-1}(S) \rightarrow H_{I}^{n-1}\left(S_{f_{1}}\right) \rightarrow H_{I+f_{1} S}^{n}(S) \rightarrow H_{I}^{n}(S) \rightarrow H_{I}^{n}\left(S_{f_{1}}\right) \rightarrow 0
$$

then implies both that $H_{I+f_{1} S}^{n-1}(S) \cong H_{I}^{n-1}(S)$, and that

$$
0 \rightarrow H_{I+f_{1} S}^{n}(S) \rightarrow H_{I}^{n}(S) \rightarrow H_{I}^{n}\left(S_{f_{1}}\right) \rightarrow 0
$$

is exact. Hence, $\operatorname{Ext}_{S}^{j}\left(K, H_{I}^{n-1}(S)\right) \cong \operatorname{Ext}_{S}^{j}\left(K, H_{I+f_{1} S}^{n-1}(S)\right)$. Moreover, $I+f_{1} S \subseteq J_{2}$ is an ideal of dimension two, whose minimal primes of dimension two are $P_{1}, \ldots, P_{r}$. If we assume that the claim is true for $\ell$, the proof for $\ell+1$ is analogous to the previous part.

Corollary 5.3. Let $(R, m, K)$ be a local ring of equal characteristic $p>0$. If $R$ is Cohen-Macaulay or if $K$ is separably closed and $\operatorname{dim}(R) \leq 2$, then for all $i, j \geq 0$, $\widetilde{\lambda}_{i, j}(R)=\lambda_{i, j}(R)$.

Proof. Since dimension, the Cohen-Macaulay property, and both types of Lyubeznik numbers are preserved under completion, we may assume that $R$ is complete. The result then follows from Propositions 5.1 and 5.2.

Question 5.4. Let $R$ be a Cohen-Macaulay local ring of dimension $d$. Kawasaki showed that $\lambda_{d, d}(R)=1$ if $R$ contains a field [13, Theorem 1]. If $R$ has mixed characteristic, is $\widetilde{\lambda}_{d, d}(R)=1$ ?

\section{An example for which the Lyubeznik numbers and the Lyubeznik numbers in mixed characteristic differ}

Remark 6.1. For a field $K$, a certain minimal triangulation of the projective plane $\mathbb{P}_{K}^{5}$ defines the Stanley-Reisner ideal of $K\left[x_{1}, \ldots, x_{6}\right]$ generated by the following ten monomials:

$$
\begin{array}{lllll}
x_{1} x_{2} x_{3} & x_{1} x_{2} x_{4} & x_{1} x_{3} x_{5} & x_{1} x_{4} x_{6} & x_{1} x_{5} x_{6} \\
x_{2} x_{3} x_{6} & x_{2} x_{4} x_{5} & x_{2} x_{5} x_{6} & x_{3} x_{4} x_{5} & x_{3} x_{4} x_{6} .
\end{array}
$$

The projective variety defined by this ideal is called Reisner's variety [23, Remark 3].

Notation 6.2. Let $R=\mathbb{Z}_{(2)}\left[x_{1}, \ldots, x_{6}\right]$ and let $I$ denote the ideal of $R$ generated by 2 and the ten monomials listed in (6.1.1). 
Under Notation 6.2, our first goal is to compute the Lyubeznik numbers in mixed characteristic of $S / I S$, where $m=\left(2, x_{1}, \ldots, x_{6}\right) R$ and $S=\widehat{R}_{m}$. Using this, we will be able to conclude that the Lyubeznik numbers in mixed characteristic need not agree with the Lyubeznik numbers for rings of equal characteristic $p>0$.

Remark 6.3. It is easily checked that for $I \subseteq R$ from Notation $6.2 \operatorname{depth}_{I}(R)=$ $\operatorname{dim}(R)-\operatorname{dim}(R / I)=7-3=4$ since $R$ is Cohen-Macaulay. With $p=2$, this means that the short exact sequence $0 \rightarrow R \rightarrow R_{p} \rightarrow R_{p} / R \rightarrow 0$ induces the long exact sequence

$$
0 \rightarrow H_{I}^{3}\left(R_{p} / R\right) \rightarrow H_{I}^{4}(R) \rightarrow H_{I}^{4}\left(R_{p}\right) \rightarrow H_{I}^{4}\left(R_{p} / R\right) \rightarrow \cdots
$$

Since $p=2 \in I, H_{I}^{i}\left(R_{p}\right)=0$ for all $i \geq 0$, so each $H_{I}^{i}\left(R_{p} / R\right) \cong H_{I}^{i+1}(R)$.

Remark 6.4. Given a polynomial $\operatorname{ring} A$ over $\mathbb{Z}$, an ideal $\mathfrak{a}$ of $A$, and a prime $p \in \mathbb{Z}$, the short exact sequence $0 \rightarrow A / p A \stackrel{\cdot p}{\rightarrow} A / p^{2} A \rightarrow A / p A \rightarrow 0$ induces the Bockstein homomorphisms $\partial_{j}: H_{\mathfrak{a}}^{j}(A / p A) \rightarrow H_{\mathfrak{a}}^{j+1}(A / p A)$ for each $j \geq 0$, the connecting homomorphisms in the long exact sequence for local cohomology. For $\mathfrak{a} \subseteq \mathbb{Z}\left[x_{1}, \ldots, x_{6}\right]$ generated by the ten monomials listed in (6.1.1), Singh and Walter showed that the Bockstein homomorphism $\partial_{3}$ is nonzero if and only if $p=2$ [24, Example 5.10].

Proposition 6.5. For $I \subseteq R$ from Notation 6.2 and $p=2$, multiplication by $p$ on $H_{I}^{3}\left(R_{p} / R\right)$ is not surjective.

Proof. Since $\operatorname{depth}_{I}(R)=4$ and each $H_{I}^{i}\left(R_{p} / R\right) \cong H_{I}^{i+1}(R), H_{I}^{i}\left(R_{p} / R\right)=0$ for $i \leq 3$ by the long exact sequence in local cohomology (see Remark 6.3). For every $\ell>0$, the exact sequence $0 \rightarrow R / p^{\ell} R \rightarrow R_{p} / R \stackrel{\cdot p^{\ell}}{\rightarrow} R_{p} / R \rightarrow 0$ induces a long exact sequence

$$
0 \rightarrow H_{I}^{3}\left(R / p^{\ell} R\right) \rightarrow H_{I}^{3}\left(R_{p} / R\right) \stackrel{\cdot p^{\ell}}{\rightarrow} H_{I}^{3}\left(R_{p} / R\right) \rightarrow H_{I}^{4}\left(R / p^{\ell} R\right) \rightarrow \cdots
$$

In particular, $H_{I}^{3}\left(R / p^{\ell} R\right) \cong \operatorname{Ann}_{H_{I}^{3}\left(R_{p} / R\right)}\left(p^{\ell} R\right)$.

As the direct limit functor is exact, the limit of the direct system of short exact sequences

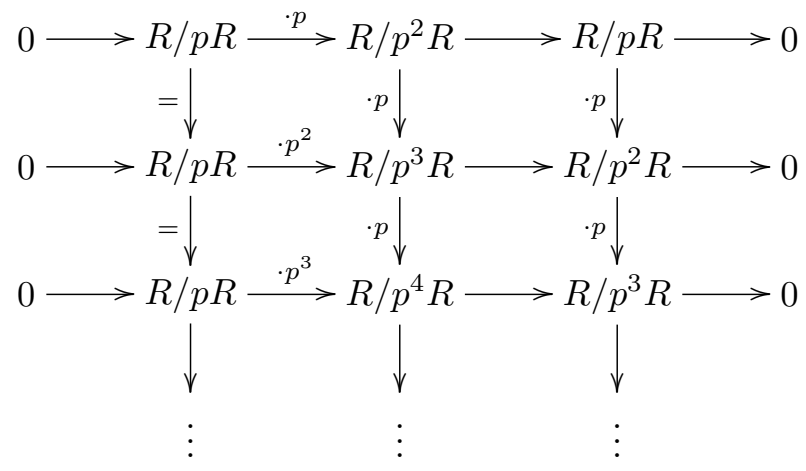


is the short exact sequence $0 \rightarrow R / p R \rightarrow R_{p} / R \stackrel{\cdot p}{\rightarrow} R_{p} / R \rightarrow 0$. Moreover, $H_{I}^{j}\left(R_{p} / R\right)=$ $\lim _{\vec{\ell}} H_{I}^{j}\left(R / p^{\ell} R\right)$. We obtain the following isomorphism of sequences.

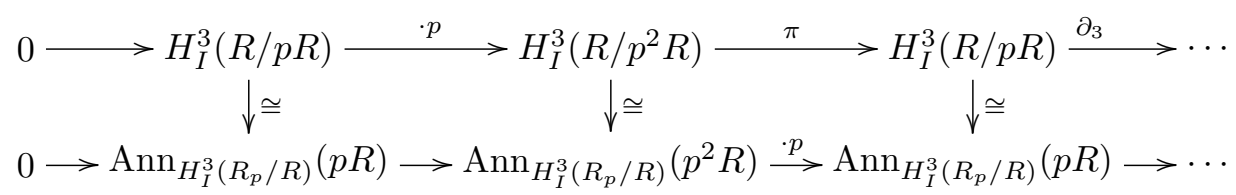

By Remark 6.4, $\partial_{3}$ is nonzero, so that $\pi$ is not surjective, and thus,

$$
\operatorname{Ann}_{H_{I}^{3}\left(R_{p} / R\right)}\left(p^{2} R\right) \stackrel{\cdot p}{\rightarrow} \operatorname{Ann}_{H_{I}^{3}\left(R_{p} / R\right)}(p R)
$$

is not surjective either. We point out that $\operatorname{Ann}_{H_{I}^{3}\left(R_{p} / R\right)}\left(p^{2} R\right)$ contains all the elements in $H_{I}^{3}\left(R_{p} / R\right)$ that map to $\operatorname{Ann}_{H_{I}^{3}\left(R_{p} / R\right)}(p R)$ under multiplication by $p$. Hence, multiplication by $p$ on $H_{I}^{3}\left(R_{p} / R\right)$ is not surjective.

Remark 6.6. Let $A=\mathbb{F}_{2}\left[y_{1}, \ldots, y_{5}\right]$, and let $J=\left(y_{1} y_{2}, y_{2} y_{3}, y_{3} y_{4}, y_{4} y_{5}, y_{5} y_{1}\right)$. Then $J=\left(y_{2}, y_{3}, y_{5}\right) \cap\left(y_{1}, y_{3}, y_{4}\right) \cap\left(y_{1}, y_{2}, y_{4}\right) \cap\left(y_{1}, y_{3}, y_{5}\right) \cap\left(y_{2}, y_{4}, y_{5}\right)$, and $A / J$ is a graded Cohen-Macaulay ring of dimension 2 , where the classes of $y_{1}+y_{2}+y_{3}$ and $y_{1}+y_{4}+y_{5}$ form a homogeneous system of parameters. Then $H_{J}^{i}(A) \neq 0$ if and only if $i=3[22$, Proposition III.4.1]. (See [1, Proposition 3.1] for an analog in characteristic zero.)

Lemma 6.7. For $I \subseteq R$ from Notation 6.2, $H_{I}^{4}(R / 2 R)$ is supported only at the maximal ideal $\left(2, x_{1}, \ldots, x_{6}\right)$.

Proof. Let $\bar{R}=R / 2 R \cong \mathbb{F}_{2}\left[x_{1}, \ldots, x_{6}\right]$. As $I \bar{R}$ is a square-free monomial ideal, by [26, Proposition 2.5] and [27, Proposition 2.7], every prime in $\operatorname{Ass}_{R} H_{I}^{4}(\bar{R})$ is of the form $\left(2, x_{i_{1}}, \ldots, x_{i_{j}}\right) R$ for some $j \geq 0$ and $1 \leq i_{1}<\cdots<i_{j} \leq 6$. Thus, it suffices to show that each $H_{I}^{4}(\bar{R})_{x_{i}}=0$.

Consider $H_{I}^{4}(\bar{R})_{x_{6}}$; the other cases are analogous. Let $A=\mathbb{F}_{2}\left[x_{1}, \ldots, x_{5}\right]$ and $J=$ $\left(x_{1} x_{2}, x_{2} x_{3}, x_{3} x_{4}, x_{4} x_{5}, x_{5} x_{1}\right) \subseteq A$. By Remark 6.6, $H_{J}^{4}(A)=0$. Since $A\left[x_{6}\right]_{x_{6}}=\bar{R}_{x_{6}}$ is a flat extension of $A, H_{J}^{4}(\bar{R})_{x_{6}}=H_{J}^{4}(A) \otimes_{A} \bar{R}_{x_{6}}=0$. As $J \bar{R}_{x_{6}}=I \bar{R}_{x_{6}}, H_{I}^{4}(\bar{R})_{x_{6}}=$ $H_{J}^{4}(\bar{R})_{x_{6}}=0$.

Corollary 6.8. Take $I \subseteq R$ from Notation 6.2, and let $S=\widehat{R}_{m}$, where $m$ is the maximal ideal $\left(2, x_{1}, \ldots, x_{6}\right)$ of $R$. Then for $p=2$,

$$
\operatorname{Coker}\left(H_{I}^{3}\left(S_{p} / S\right) \stackrel{\cdot p}{\rightarrow} H_{I}^{3}\left(S_{p} / S\right)\right) \cong H_{I}^{4}(S / p S) \cong E_{S / p S}\left(\mathbb{F}_{2}\right) .
$$

Proof. Note that $S=\widehat{\mathbb{Z}}_{(2)} \llbracket x_{1}, \ldots, x_{6} \rrbracket$, where $\widehat{\mathbb{Z}}_{(2)}$ denotes the 2-adic integers; moreover, $S / p S \cong \mathbb{F}_{2} \llbracket x_{1}, \ldots, x_{6} \rrbracket$. By Lemma $6.7, H_{I}^{4}(S / p S)$ is supported only at $m$, so $H_{I}^{4}(S / p S)=H_{m}^{0} H_{I}^{4}(S / p S)$, and, thus, is injective by [15, Corollary 3.6]; as its Bass numbers are finite [12, Theorem 2.1], $H_{I}^{4}(S / p S) \cong \bigoplus_{i=1}^{\alpha} E_{S / p S}\left(\mathbb{F}_{2}\right)$ for some $\alpha \geq 0$. By a calculation in [2, Example 4.8] (see Remark 6.11), $\operatorname{dim}_{\mathbb{F}_{2}} \operatorname{Hom}_{\mathbb{F}_{2}}\left(\mathbb{F}_{2}, H_{I}^{4}(S)\right)=$ $\lambda_{0,2}(S / p S)=1$, so that $\alpha=1$ and $H_{I}^{4}(S / p S) \cong E_{S / 2 S}\left(\mathbb{F}_{2}\right)$.

By the long exact sequence

$$
0 \rightarrow H_{I}^{3}(S / p S) \rightarrow H_{I}^{3}\left(S_{p} / S\right) \stackrel{\cdot p}{\rightarrow} H_{I}^{3}\left(S_{p} / S\right) \rightarrow H_{I}^{4}(S / p S) \rightarrow \cdots
$$


induced by $0 \rightarrow S / p S \rightarrow S_{p} / S \stackrel{\cdot p}{\rightarrow} S_{p} / S \rightarrow 0$, Coker $\left(H_{I}^{3}\left(S_{p} / S\right) \stackrel{\cdot p}{\rightarrow} H_{I}^{3}\left(S_{p} / S\right)\right)$ injects into $H_{I}^{4}(S / p S)=E_{S / p S}\left(\mathbb{F}_{2}\right)$. Thus, this cokernel is a $D\left(S / p S, \mathbb{F}_{2}\right)$-submodule of $E_{S / p S}\left(\mathbb{F}_{2}\right)$, itself a simple $D\left(S / p S, \mathbb{F}_{2}\right)$-module. Since it is nonzero by Proposition 6.5, we are done.

Corollary 6.9. There exists an unramified regular local ring $S$ of mixed characteristic $p=2$, and an ideal $I$ of $S$ containing $p$, such that multiplication by $p$ on $H_{I}^{4}(S)$ is not surjective.

Proof. Take $I \subseteq R$ from Notation 6.2 , let $m=\left(2, x_{1}, \ldots, x_{6}\right) R$, and let $S=\widehat{R}_{m}$. Then by Corollary 6.8, noting Remark 6.3, Coker $\left(H_{I}^{4}(S) \stackrel{\cdot p}{\rightarrow} H_{I}^{4}(S)\right) \cong E_{S / p S}\left(\mathbb{F}_{2}\right) \neq 0$.

Proposition 6.10. Take $I \subseteq R$ from Notation 6.2, and let $S=\widehat{R}_{m}$, where $m=$ $\left(2, x_{1}, \ldots, x_{6}\right)$. Then $\tilde{\lambda}_{i, j}(S / I S)=1$ if $i=j=3$, and vanishes otherwise.

Proof. For brevity, let $I$ denote $I S$, and let $p=2$. In [14, Theorem 1 and Example 1], it is shown that $H_{I}^{5}(S / p S)=0$ (relying on the fact that $S / p S$ has characteristic two). By Corollary 6.8 and Remark 6.3 , the short exact sequence $0 \rightarrow S \stackrel{\cdot p}{\rightarrow} S \rightarrow S / p S \rightarrow 0$ then gives rise to the long exact sequence

$$
\ldots \rightarrow H_{I}^{4}(S) \stackrel{\cdot p}{\rightarrow} H_{I}^{4}(S) \rightarrow H_{I}^{4}(S / p S) \stackrel{0}{\rightarrow} H_{I}^{5}(S) \stackrel{\cdot p}{\rightarrow} H_{I}^{5}(S) \rightarrow 0 \rightarrow H_{I}^{6}(S) \stackrel{\cdot p}{\rightarrow} \ldots
$$

Thus, multiplication by $p$ on $H_{I}^{5}(S)$ and on $H_{I}^{6}(S)$ are injective maps, which implies that $H_{I}^{5}(S)=H_{I}^{6}(S)=0$ since $p \in I$. Moreover, $H_{I}^{i}(S)=0$ for $i \geq 6$ as well, so by again noting Corollary 6.8, $H_{I}^{i}(S) \neq 0$ if and only if $i=4$.

This means that the spectral sequence $E_{2}^{p, q}=H_{m}^{p} H_{I}^{q}(S) \underset{p}{\Longrightarrow} H_{m}^{p+q}(S)=E_{\infty}^{p, q}$ converges at the second stage. Thus, $H_{m}^{3} H_{I}^{4}(S) \cong H_{m}^{7}(S) \cong E_{S}\left(\mathbb{F}_{2}\right)$, and all other $H_{m}^{p} H_{I}^{q}(S)$ vanish. Since all $H_{m}^{p} H_{I}^{q}(S)$ are injective $S$-modules, [15, Lemma 1.4] implies that the Bass number $\operatorname{dim}_{\mathbb{F}_{2}} \operatorname{Ext}_{S}^{p}\left(\mathbb{F}_{2}, H_{I}^{q}(S)\right)$ equals $\operatorname{dim}_{\mathbb{F}_{2}} \operatorname{Hom}_{\mathbb{F}_{2}}\left(\mathbb{F}_{2}, H_{m}^{p} H_{I}^{q}(S)\right)$ for all $p, q \geq 0$. Since $\operatorname{dim}(R)=7, \widetilde{\lambda}_{i, j}(R)=\operatorname{dim}_{K} \operatorname{Hom}_{S}\left(\mathbb{F}_{2}, H_{m}^{i} H_{I}^{7-j}(S)\right)=1$ if $i=j=$ 3 , and vanishes otherwise.

Remark 6.11. Finally, we conclude that the two notions of Lyubeznik numbers do not always agree when both are defined. Take $I \subseteq R$ as defined in Notation 6.2. Let $S_{1}=\mathbb{F}_{2} \llbracket x_{1}, \ldots, x_{6} \rrbracket$ and $S_{2}=\widehat{\mathbb{Z}}_{(2)} \llbracket x_{1}, \ldots, x_{6} \rrbracket$. Àlvarez Montaner and Vahidi found $\Lambda\left(S_{1} / I S_{1}\right)$ [2, Example 4.8], and we have have calculated $\widetilde{\Lambda}\left(S_{2} / I S_{2}\right)$, relying on Remark 6.4. The tables are as follows:

$$
\Lambda\left(S_{1} / I S_{1}\right)=\left(\begin{array}{cccc}
0 & 0 & 1 & 0 \\
0 & 0 & 0 & 0 \\
0 & 0 & 0 & 1 \\
0 & 0 & 0 & 1
\end{array}\right) \quad \text { and } \quad \widetilde{\Lambda}\left(S_{2} / I S_{2}\right)=\left(\begin{array}{cccc}
0 & 0 & 0 & 0 \\
0 & 0 & 0 & 0 \\
0 & 0 & 0 & 0 \\
0 & 0 & 0 & 1
\end{array}\right) .
$$

This means that if $A$ is the completion of the Stanley Reisner ring of the ideal in Remark 6.1 with $K=\mathbb{F}_{2}$, then $\lambda_{0,2}(A)=\lambda_{2,3}(A)=1$, while $\widetilde{\lambda}_{0,2}(A)=\widetilde{\lambda}_{2,3}(A)=0$. In particular, this gives a negative answer to Question 3.9.

We note that when $K$ has characteristic zero, the Lyubeznik table of the monomial ideal in Remark 6.1 that inspired this calculation coincides with $\widetilde{\Lambda}\left(S_{2} / I S_{2}\right)$ 
[2, Example 4.8]. We also note that the computations in this remark are related to work in [1].

Theorem 6.12. There exists an unramified regular local ring $(S, m, K)$ of mixed characteristic $p=2$, and an ideal $I$ of $S$, such that for some $i, j \geq 0$,

$$
\operatorname{dim}_{K} \operatorname{Ext}_{S}^{j}\left(K, H_{I}^{i}(S)\right) \neq \operatorname{dim}_{K} \operatorname{Ext}_{S / p S}^{j}\left(K, H_{I S / p S}^{i-1}(S / p S)\right)
$$

Proof. Take $I \subseteq R$ from Notation 6.2 , and let $S=\widehat{R}_{m}$, where $m=\left(2, x_{1}, \ldots, x_{6}\right)$. Then $\operatorname{dim}_{K} \operatorname{Hom}_{R}\left(K, H_{I}^{5}(S)\right)=0 \neq 1=\operatorname{dim}_{K} \operatorname{Hom}_{S / p S}\left(K, H_{I S / p S}^{4}(S / p S)\right)$ by Proposition 6.10 and Remark 6.11.

Question 6.13. Suppose that $R$ is local ring of equal characteristic $p>0$ and of dimension $d$. Is $\widetilde{\lambda}_{d, d}(R)=\lambda_{d, d}(R)$ ?

\section{Acknowledgments}

We are grateful to Josep Àlvarez Montaner, Daniel J. Hernández, Mel Hochster, Youngsu Kim, Gennady Lyubeznik, and Felipe Pérez for their valuable comments and helpful discussions. In addition, we thank Gennady Lyubeznik for also noticing a key point we initially overlooked regarding Theorem 3.5. We thank the 2010 AMS Mathematical Research Communities program for supporting meetings between the authors. The first author also thanks the National Council of Science and Technology of Mexico for support through Grant 210916.

\section{References}

[1] J. Àlvarez Montaner, R. García López and S. Zarzuela Armengou, Local cohomology, arrangements of subspaces and monomial ideals, Adv. Math. 174(1) (2003), 35-56.

[2] J. Àlvarez Montaner and A. Vahidi, Lyubeznik numbers of monomial ideals, Trans. Amer. Math. Soc. 366(4) (2014), 1829-1855.

[3] J.-E. Björk, The global homological dimension of some algebras of differential operators, Invent. Math. 17 (1972), 67-78.

[4] M. Blickle and R. Bondu, Local cohomology multiplicities in terms of étale cohomology, Ann. Inst. Fourier (Grenoble) 55(7) (2005), 2239-2256.

[5] I.S. Cohen, On the structure and ideal theory of complete local rings, Trans. Amer. Math. Soc. 59 (1946), 54-106.

[6] S.C. Coutinho, A primer of algebraic D-modules, Vol. 33 of London Mathematical Society Student Texts, Cambridge University Press, Cambridge (1995), ISBN 0-521-55119-6; 0-52155908-1.

[7] R. García López and C. Sabbah, Topological computation of local cohomology multiplicities, Collect. Math. 49(2-3) (1998), 317-324. Dedicated to the memory of Fernando Serrano.

[8] A. Grothendieck, Éléments de géométrie algébrique. IV. Étude locale des schémas et des morphismes de schémas IV, Inst. Hautes Études Sci. Publ. Math. 32 (1967), 361.

[9] R. Hartshorne, Local cohomology, Vol. 1961 of A seminar given by A. Grothendieck, Harvard University, Fall, Springer-Verlag, Berlin (1967).

[10] - Cohomological dimension of algebraic varieties, Ann. Math. (2) 88 (1968), 403-450.

[11] C. Huneke and G. Lyubeznik, On the vanishing of local cohomology modules, Invent. Math. 102(1) (1990), 73-93.

[12] C.L. Huneke and R.Y. Sharp, Bass numbers of local cohomology modules, Trans. Amer. Math. Soc. 339(2) (1993), 765-779.

[13] K.-i. Kawasaki, On the highest Lyubeznik number, Math. Proc. Cambridge Phil. Soc. 132(3) (2002), 409-417. 
[14] G. Lyubeznik, On the local cohomology modules $H_{\mathfrak{a}}^{i}(R)$ for ideals a generated by monomials in an R-sequence, in Complete intersections (Acireale, 1983), Vol. 1092 of Lecture Notes in Math., 214-220, Springer, Berlin (1984).

[15] - Finiteness properties of local cohomology modules (an application of D-modules to commutative algebra), Invent. Math. 113(1) (1993), 41-55.

[16] - Finiteness properties of local cohomology modules: a characteristic-free approach, J. Pure Appl. Algebra 151(1) (2000), 43-50.

[17] - Finiteness properties of local cohomology modules for regular local rings of mixed characteristic: the unramified case, Comm. Algebra 28(12) (2000), 5867-5882. Special issue in honor of Robin Hartshorne.

[18] - Injective dimension of D-modules: a characteristic-free approach, J. Pure Appl. Algebra 149(2) (2000), 205-212.

[19] - On some local cohomology invariants of local rings, Math. Z. 254(3) (2006), 627-640.

[20] L. Núñez-Betancourt, Local cohomology modules of polynomial or power series rings over rings of small dimension, arXiv:1207.1896, to appear in Illinois J. Math. (2014).

[21] L. Núñez-Betancourt and E.E. Witt, Generalized Lyubeznik numbers, arXiv:1208:5500, to appear in Nagoya Math. J. 215 (2014).

[22] C. Peskine and L. Szpiro, Dimension projective finie et cohomologie locale. Applications à la démonstration de conjectures de M. Auslander, H. Bass et A. Grothendieck, Inst. Hautes Études Sci. Publ. Math. 42 (1973), 47-119.

[23] G.A. Reisner, Cohen-Macaulay quotients of polynomial rings, Advances in Math. 21(1) (1976), $30-49$.

[24] A.K. Singh and U. Walther, Bockstein homomorphisms in local cohomology, J. Reine Angew. Math. 655 (2011), 147-164.

[25] U. Walther, On the Lyubeznik numbers of a local ring, Proc. Amer. Math. Soc. 129(6) (2001), 1631-1634 (electronic).

[26] K. Yanagawa, Alexander duality for Stanley--Reisner rings and squarefree $\mathbf{N}^{n}$-graded modules, J. Algebra 225(2) (2000), 630-645.

[27] - Bass numbers of local cohomology modules with supports in monomial ideals, Math. Proc. Cambridge Phil. Soc. 131(1) (2001), 45-60.

[28] W. Zhang, On the highest Lyubeznik number of a local ring, Compos. Math. 143(1) (2007), 82-88.

[29] C. Zhou, Higher derivations and local cohomology modules, J. Algebra 201(2) (1998), 363-372.

Department of Mathematics, University of Virginia, Charlottsville, VA 22904, USA

E-mail address: 1cn8m@virginia.edu

Department of Mathematics, University of Minnesota, Minneapolis, MN 55455, USA

E-mail address: ewitt@umn.edu 
